\title{
Implication of RNA splicing in heat stress responses and expression of a novel trypsin-like protease in the marine diatom Chaetoceros compressum
}

\author{
SHIGEHARU KINOSHITA, ${ }^{1}$ HIROSHI YAMADA, ${ }^{2}$ TAKEYA HARA, ${ }^{2}$ YASUO ITOH ${ }^{2}$ AND SHUGO \\ WATABE $^{1 *}$ \\ ${ }^{1}$ Laboratory of Aquatic Molecular Biology and Biotechnology, Graduate School of Agricultural and Life Sciences, \\ The University of Tokyo, Tokyo 113-8657, Japan (awatabe@mail.ecc.u-tokyo.ac.jp) and ${ }^{2}$ Central Laboratory \\ Marine Ecology Research Institute, Chiba 299-5105, Japan
}

SUMMARY: One explanation for the predominance of diatoms in marine ecosystem is their high adaptability to different environmental conditions. However, little is known about the physiological mechanisms concerned at the molecular level. Recent works on heat stress responses of various diatom species have indicated that diatoms markedly change their gene expression profiles and produce various molecules to respond to heat stress. Recently, we have isolated a novel heat-stress-responsive gene, $\mathrm{HI}-5$, from the marine diatom Chaetoceros compressum. Two types of transcripts were produced from the single HI-5 gene by heat stress-dependent RNA splicing, encoding structurally different proteins, the functions of which are apparently different. This gene may be a promising molecule to give us a new insight for better understanding of heat stress responses in diatoms.

\section{KEY WORDS: alternative RNA splicing, diatom, heat stress, serine protease}

\section{INTRODUCTION}

Diatoms are the most abundant phytoplankton and are situate at an extremely important primary production niche in aquatic ecosystem. They also play a key role in biogeochemical cycling of minerals and global carbon fixation, distributing from tropical to polar regions. Some diatom species live even under sever conditions such as those in high acidity and high alkalinity. One explanation for such a diverse inhabitation of diatoms is their high adaptability to different environmental conditions. ${ }^{1)} \quad$ However, little is known about the physiological mechanisms involved.

Heat stress is one of the most common stresses for organisms in aquatic environment. The most well-known reaction against heat stress is the production of highly conserved heat shock proteins (HSPs). These prevent aggregation of heat-denatured proteins and facilitate the refolding of nascent proteins. ${ }^{2)}$ They also play important roles in cell growth, development, and differentiation under nomal conditions. Diatoms seem to have similar stress response systems, because the expression of various HSPs in the diatoms Odontella weissflogii ${ }^{3)}$ and Skeletonema coastatum ${ }^{4,5)}$ upon exposure to heat stress has been reported at the translational level.

Protein degradation is the other essential event to protect cells under heat stress conditions. Ubiquitin, one of the obligatory proteins, is highly conserved, and its conjugated proteins are specifically proteolyzed by the proteasome. Heat shock accelerates the accumulation of ubiquitinated proteins in diatom $S$. costatum cells. ${ }^{4,5}$

In plant cells, the change in polyamine content in response to a variety of stress conditions has been also investigated. $^{6)}$ Polyamine is ubiquitous to plant cells and known to be involved in DNA replication, cell differentiation, and growth regulation. Scoccianti et al. ${ }^{5)}$ demonstrated that putrescine and spermidine increase after heat stress in the diatom $S$. costatum cells. A protective effect on membrane integrity or the stabilization of proteins by inhibition of degradative enzymes has been proposed for these polyamines.

The glutamine synthetase gene is also expressed in the diatom Chaetoceros compressum cells under heat stress conditions. ${ }^{\text {) }}$ The induction of this gene by heat stress is reasonable, because glutamine induces the expression of HSPs in various organisms. ${ }^{8-12)}$

Such observation suggests that diatoms dramatically alter their gene expression profiles in response to heat stress. These heat stress-responsive genes may have bestowed diatoms their high adaptability to various environmental conditions. Recently, we have isolated a novel heat stress-responsive gene from the marine diatom C. compressum. ${ }^{13)}$ The expression of one gene, named $\mathrm{HI}-5$, was induced by heat stress through a unique, but complex, regulation system

In this review, we consider heat stress response of 
diatoms on the basis of our recent investigations, showing a novel candidate gene involved in their response.

\section{HI-5, A HEAT RESPONSIVE GENE, IN THE MARINE DIATOM}

The HI-5 gene from the marine diatom $C$. compressum consists of four exons and three introns and is transcribed into two types of mRNAs by heat stress-dependent RNA splicing (Fig. 1). One transcript, HI-5a mRNA, contains four exons and quickly increases mRNA levels following heat stress. It has an open reading frame (ORF) of 1,281 nucleotides (nt) encoding a protease domain characteristic to trypsin-like serine proteases (Fig. 1). The other transcript, HI-5b mRNA, retains the second intron in addition to four exons described above and is constitutively expressed in contrast to HI-5a mRNA. HI-5b mRNA contains ORF of only 933 nt due to the presence of the stop codon in the second intron and encodes a protein, which partially lacks the protease domain and a C-terminal region contained in $\mathrm{H}-5 \mathrm{a}$ (Fig. 1).

The accumulation of denatured proteins by heat stress is lethal to cells. These denatured proteins are refolded by molecular chaperones or degraded by heat stress-induced proteases. ${ }^{14)}$ Proteases also function in cell signaling under heat stress conditions. For example, ATF6, a mammalian transcription factor, is activated by the proteolysis upon heat stress and enhances the expression of molecular chaperones. ${ }^{15}$ The other physiological event induced by the proteolysis under heat stress conditions is apoptosis. Severe heat stress recruits the apoptosis of cells and cysteine proteases called caspase are primarily involved in this process. ${ }^{16}$ ) A caspase-independent apoptosis has been also reported, ${ }^{17)}$ where trypsin-like serine proteases function instead of caspases. However, the induction of apoptosis with increasing protease activity has not been directly demonstrated in diatom cells. ${ }^{18)} \quad \mathrm{HI}-5 \mathrm{a}$ involved in heat stress responses in diatom cells possibly has protease activity.

\section{A SUPPOSED FAIL-SAFE MECHANISM FOR REGULATORY EXPRESSION OF THE HI-5 GENE PRODUCTS}

HI-5b mRNA is produced by heat stress-dependent RNA splicing from the HI-5 gene as described before. However, its encoding protein lacks a trypsin-like serine protease domain, suggesting that it has no proteolytic activity. Why is this apparently incomplete molecule produced in diatom cells? One possibility is that HI-5b mRNA is accumulated as a premature form of HI-5a mRNA. In this case, heat stress may induce RNA
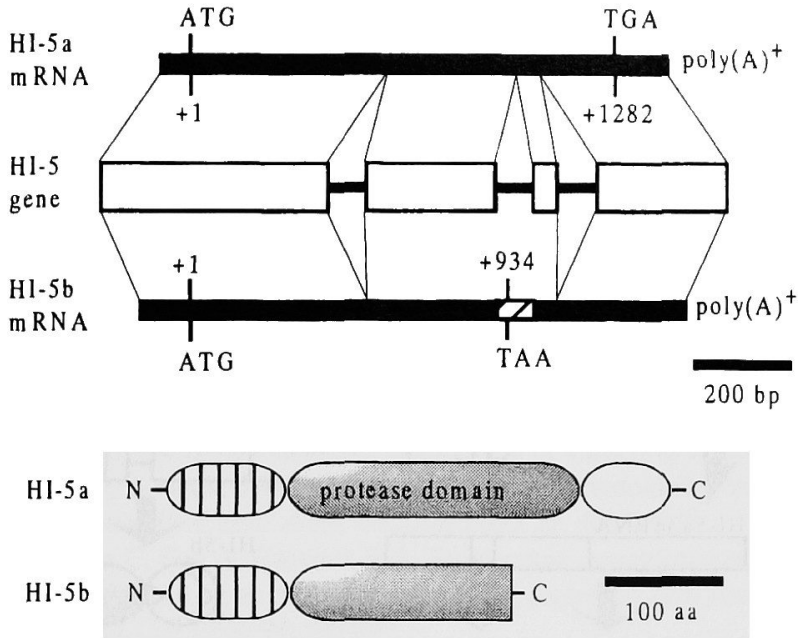

Figure 1. Schematic diagram of HI-5a and HI-5b mRNAs transcribed from the HI-5 gene and their translated product. The HI-5 gene consists of 4 exons (open box) and 3 introns (solid line). ${ }^{13)} \quad$ The inserted sequence (shaded box) in HI-5b mRNA is encoded by the second intron. The numbers represent nucleotides from adenine at the start codon. HI-5a mRNA has the stop codon at 1,282-1,284 nt (TGA), whereas HI-5b mRNA does at $934-936$ nt (TAA) near the $5^{\prime}$ end of an inserted sequence. While HI-5a contains protease domain characteristic to typsin-like serine proteases, HI- $5 \mathrm{~b}$ has an incomplete protease domain where the S1 binding pocket and residues around active serine are truncated. The additional sequence of HI-5a following the protease domain does not exist in HI-5b.

splicing to produce immediately HI-5a mRNAs from accumulated HI-5b mRNAs (model 1 in Fig. 2). It is noted that similar phenomena have been reported for Hac1, a transcription factor acting on the genes which encode molecular chaperones of the yeast Saccharomyces cerevisiae. $^{19)}$ Hacl mRNA is constitutively expressed as a precursor form incorporating an intron under normal conditions. Various kinds of stress including those caused by heat induced RNA splicing on Hac1 mRNA, resulting in the formation of mature mRNAs with no intron. Interestingly, an artificially expressed $\mathrm{Hac1}$ homologue from precursor mRNAs shows much weaker transcription activity than the genuine Hacl from mature mRNAs. ${ }^{20)}$ This is so-called a fail-safe mechanism to prevent misleading production of mature mRNAs under normal conditions. Diatom cells may have acquired the second intron to be transcribed from the HI-5 gene in a similar fail-safe mechanism during evolutionary time span to prevent redundant expression of unnecessary proteases under normal conditions.

Another possibility is that $\mathrm{HI}-5 \mathrm{~b}$ has activity different from protease (model 2 in Fig. 2). In this case, HI-5a with proteolytic activity will be produced only when cells are exposed to heat stress. Most proteases involved in stress response have chaperone activity in addition to their inherent proteolytic activity. DegP, a stress-induced 


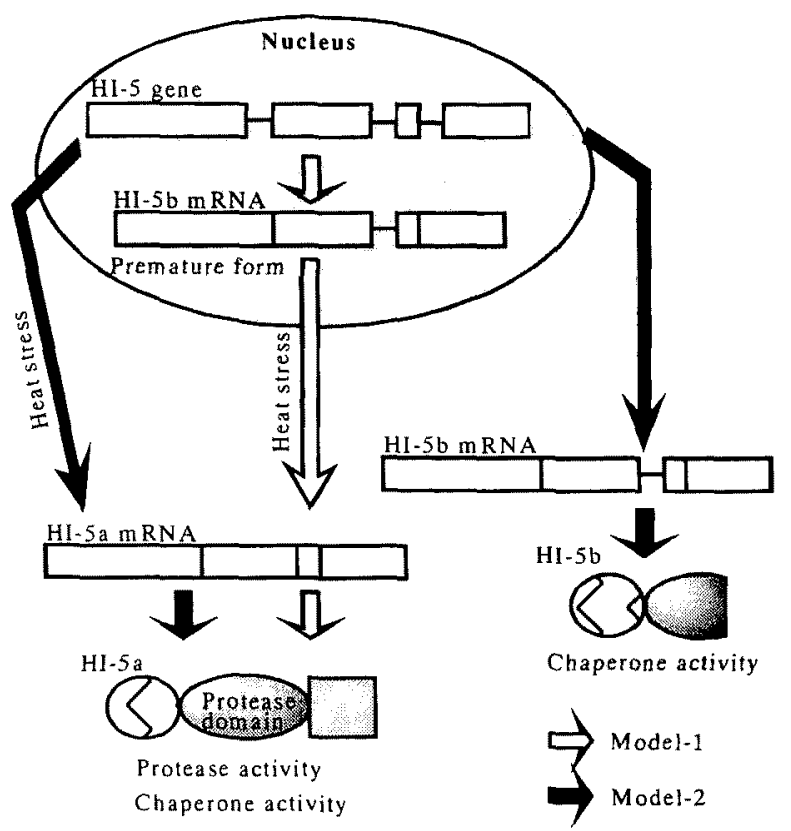

Figure 2. Heat stress-dependent expression of the $H I-5$ gene products and their passible activities. Two possible models for regulation of the $H I-5$ gene expression are represented. In model $1, \mathrm{HI}-5 \mathrm{~b}$ mRNA exists as a premature form of HI-5a mRNA. Heat stress activates RNA splicing to produce $\mathrm{HI}-5 \mathrm{a}$ mRNA. In model $2, \mathrm{HI}-5 \mathrm{~b}$ has different activity from protease, i.e., chaperon activity under normal conditions. When cells are exposed to heat stress, HI-5a with protease activity is produced.

serine protease in Escherichia coli, functions as a molecular chaperone under normal conditions and as a proteolytic enzyme under stress conditions. ${ }^{21)} \mathrm{FtsH}$, Yta10/Yta12 and Piml/Lon are other stress-induced proteases and also have exherent chaperon activity. ${ }^{2-24)}$ It is noted that these proteases maintain chaperone activity even when their protease domains are truncated. ${ }^{25}$

\section{TRANSCRIPTIONAL VARIATION INTRODUCED BY RNA SPLICING}

The above observation with the HI-5 gene is the first finding for gene expression regulated by altemative splicing in diatoms. Transcriptional variation introduced by RNA splicing seems to be common to many organisms. The alignment of EST sequences in the human genome project has recently disclosed that at least $35 \%$ of human genes produce variably spliced transcripts. ${ }^{26)}$ There are also many examples which showed divergent mRNAs produced from a single gene. $^{27)}$ However, the regulatory mechanisms involved in alternative splicing are poorly understood. A number of regulatory proteins will bind to splice sites in a precursor mRNA. ${ }^{27}$ These proteins function as positive and negative factors regulating RNA splicing to produce appropriate mRNAs. So far, only a few regulatory proteins have been identified.
Heat stress also regulates alternative splicing and the HI-5 gene is one of the typical examples. Yost and Lindquist ${ }^{28)}$ claimed that severe heat stress prevented the splicing of introns from HSP83 mRNA precursors in the fruit fly Drosophila melanogaster cells. However, heat stress on diatom cells rather accelerated the splicing of the HI-5 gene. It seems that there is a certain heat stress-dependent splicing factor(s), which binds specifically to the HI-5 precursor mRNAs and facilitate its splicing. Three introns of the HI-5 gene are of almost the same size, namely 91, 112 and 107 nt. The introns have a short consensus sequence specific for RNA splicing at 5'- and 3'-splice site and contained an additional element called the branch site. However, it is not clear why the second intron of the HI-5 gene is not spliced specifically.

\section{CONCLUSION}

In spite of the ecological importance of diatoms in the aquatic environment, very little molecular biological studies have been attempted. Our study on the HI-5 gene will give a new insight for better understanding of heat stress responses in diatoms at the molecular level. However, physiological functions of our proteins in diatom cells are still unclear. Further study is now in progress to elucidate functions of the $H I-5$ gene products produced under normal conditions and in heat stress responses. Furthermore, the recent development for generating transgenic diatoms ${ }^{29)}$ will be also helpful in our future investigations.

\section{ACKNOWLEDGMENTS}

The diatom $C$. compressum cells were kindly provided by T. Sawaguchi, the Environmental Analysis Department, Japan NUS Co., Ltd. We express sincere thanks to Dr. $\mathrm{K}$ Furuya, Laboratory of Aquatic Biology and Environmental Science, Graduate School of Agricultural and Life Sciences, The University of Tokyo, for his kind advise in cell culture. This work was supported in part by Grant-in-Aids for Scientific Research from the Stee] Industry Foundation for the Advancement of Environmental Protection Technology, and from the Ministry of Education, Culture, Sports, Science and Technology of Japan. S. K. was supported by the Research Fellowship of the Japan Society for the Promotion of Science for Young Scientists.

\section{REFERENCES}

1. Bonin DJ, Droop MR, Maestrini SY, Bonin MC. Physiological features of six micro-algae to be used as indicators of seawater quality. Crypt Algol. 1986; 7: 23-83.

2. Ellis RJ, van der Vies SM. Molecular chaperones. Annu. Rev. 
Biochem. 1991; 60: 321-347.

3. Döhler G, Hoffmann M, Stappel U. Pattern of proteins after heat shock and UV-B radiation of some temperate marine diatoms and the Antarctic Odontella weissflogii. Bot. Acta 1995; 108: 93-98.

4. Penna A. Modulation of the heat shock ubiquitin pool in Skeletonema costatum Bacillariophyceae. J. Phycol. 1996; 32: 409-415.

5. Scoccianti V, Penna A, Penna N, Magnani M. Effect of heat stress on polyamine content and protein pattern in Skeletonema costatum. Mar. Biol. 1995; 121: 549-554.

6. Galston AW. Polyamines and plant response to stress. In: Bachrach U, Heimer YN (eds). The physiology of polyamines. CRC press, Florida. 1989; 99-106.

7. Kinoshita S, Kikuchi K, Yamada H, Hara T, Itoh Y, Kinoshita H, Watabe S. Differential expression of mRNAs in the marine diatom Chaetoceros compressum exposed to high temperatures. Fisheries Sci. 1998; 64: 831-835.

8. Cai JW, Hughes CS, Shen JW, Subjeck JR. Induction of heat-shock proteins by glutamine. The 'feeding effect'. FEBS Lett. 1991; 2: 229-232.

9. Chow A, Zhang R. Glutamine reduces heat shock-induced cell death in rat epithelial cells. J. Nutr. 1998; 128: 1296-1301.

10. Nissim I, States B, Hardy M, Pleasure J. Effect of glutamine on heat-shock-induced mRNA and stress proteins. I. Cell Physiol. 1993; 157: 313-318.

11. Sanders MM, Kon C. Glutamine is a powerful effector of heat shock protein expression in Drosophila Kc clls.J. Cell. Physiol. 1991; 146: 180-190.

12. Zhou $\mathrm{X}$, Thompson JR. Regulation of protein turnover by glutamine in heat-shocked skeletal myotubes. Biochim. Biophys. Acta 1997; 1357: 234242.

13. Kinoshita S, Kaneko G, Lee JH, Yamada H, Hara T, Itoh Y, Watabe $S$. A novel heat stress-responsive gene in the marine diatom Chaetoceros compressum encoding two types of transcripts, a trypsin-like protease and its related protein, by alternative RNA splicing. Eur. J. Biochem. 2001; 268: 4599-4609.

14. Gottesman S, Wickner S, Maurizi MR Protein quality control: triage by chaperones and proteases. Genes Dev. 1997; 11: $815 \cdot 823$.

15. Haze K, Yoshida H, Yanagi H, Yura T, Mori K. Mammalian transcription factor ATF6 is synthesized as a transmembrane protein and activated proteolysis in response to endoplasmic reticlum stress. Mol Biol. Cell 1999; 10: 3787-3799.
16. Budd RC. Activation-induced cell death. Cum. Opin. Immunol. $2001 ; 13: 356-362$.

17. Adjei PN, Kaufmann SH, Leung WY, Mao F, Gores GJ. Selective induction of apoptosis in Hep 3B cells by topoisomerase I inhibitors: evidence for a protease-dependent pathway that does not activate cysteine protease P32. J. Clin. Invest. 1996; 98: 2588-2596.

18. Berges JA, Fallkowski PJ. Physiological stress and cell death in marine phytoplankton: induction of proteases in response to nitrogen or light limitation. Limnol. Oceanogr. 1998; 43: 129-135.

19. Mori K, Ogawa N, Kawahara T, Yanagi H, Yura T. mRNA splicing-mediated C-terminal replacement of transcription factor Haclp is required for efficient activation of the unfolded protein response. Proc. Nath Acad Sci. USA 2000; 25: 4660-4665.

20. Spiess C, Beil A, Ehrmann MA. Temperature-dependent switch from chaperone to protease in a widely conserved heat shock protein. Cell 1999; 97: 339-347.

21. Horwich AL. Molecular chaperones. Resurrection or destruction? Curr: Biol. 1995; 5: 455-458.

22. Langer $T$, Neupert $W$. Regulated protein degradation in mitochondria. Experientia 1996; 52: 1069-1076.

23. Schirmer EC, Glover MA, Lindquist S. HSP100/Clp proteins: a common mechanism explains diverse functions Trends Biochem Sci. 1996; 21: 289-296.

24. Arlt $\mathrm{H}$, Tauer $\mathrm{R}$, Feldmann $\mathrm{H}$, Neupert $\mathrm{W}$, Langer $\mathrm{T}$. The YTA10-12 complex, an AAA protease with chaperone-like activity in the inner membrane of mitochondria. Cell 1996; 85 : 875-885.

25. Rep M, van Dijl JM, Suda K, Schatz G, Grivell LA, Suzuki CK. Promotion of mitochondnial membrane complex assembly by a proteolytically inactive yeast Lon. Science 1996; 274: 103-106.

26. Croft L, Schandorff S, Clark F, Burrage K, Arctander P, Mattick JS. ISIS, the intron information system, reveals the high frequency of alternative splicing in the human genome. Nature Genet. 2000; 24: 340-341.

27. Black DL. Protein diversity from altemative splicing: a challenge for bioinformatics and post-genome biology. Cell 2000; 103: 367-370.

28. Yost HJ, Lidquist $\mathrm{S}$. Translation of unspliced transcripts after heat shock. Science 1988; 242: 1544-1548.

29. Falciatore A, d'Alcalá MR, Croot P, Bowler C. Perception of environmental signals by a marine diatom. Science $2000 ; 288$ : 2363-2366. 